Check for updates

Cite this: Phys. Chem. Chem. Phys., 2019, 21, 17001

Received 31st May 2019

Accepted 19th July 2019

DOI: 10.1039/c9cp03099a

rsc.li/pccp

\title{
The local electron attachment energy and the electrostatic potential as descriptors of surface-adsorbate interactions $\dagger$
}

\begin{abstract}
Joakim Halldin Stenlid, (D) *ab Adam Johannes Johansson (D) ${ }^{c}$ and Tore Brinck (D) *a
Two local reactivity descriptors computed by Kohn-Sham density functional theory (DFT) are used to predict and rationalize interactions of nucleophilic molecules (exemplified by $\mathrm{CO}$ and $\mathrm{H}_{2} \mathrm{O}$ ) with transition metal (TM) and oxide surfaces. The descriptors are the electrostatic potential, $V_{S}(\mathbf{r})$, and the local electron attachment energy, $E_{S}(\mathbf{r})$, evaluated on surfaces defined by the $0.001 \mathrm{e} \mathrm{Bohr}^{-3}$ isodensity contour. These descriptors have previously shown excellent abilities to predict regioselectivity and rank molecular as well as nanoparticle reactivities and interaction affinities. In this study, we generalize the descriptors to fit into the framework of periodic DFT computations. We also demonstrate their capabilities to predict local surface propensity for interaction with Lewis bases. It is shown that $E_{\mathrm{S}}(\boldsymbol{r})$ and $V_{\mathrm{S}}(\boldsymbol{r})$ can rationalize the interaction behavior of TM oxides and of fCC TM surfaces, including low-index, stepped and kinked surfaces spanning a wide range of interaction sites with varied coordination environments. Broad future applicability in surface science is envisaged for the descriptors, including heterogeneous catalysis and electrochemistry.
\end{abstract}

\section{Introduction}

The ability to predict and rationalize the interactions and reactions of extended surfaces is of great interest in numerous research fields spanning from heterogeneous catalysis, electrochemistry and corrosion, to nanotoxicity, biocompatibility and biodegradability. In the present paper we discuss an alternative direction to assess surface reactivity as compared to those traditionally used in the materials science and surface chemistry communities. We will show that two local properties, the local electron attachment energy $[E(\mathbf{r})]$ and the electrostatic potential $[V(\mathbf{r})]$, can be used for characterization of site-resolved Lewis acidity of transition metal and oxide surfaces when evaluated on contours of constant electron density, i.e. isodensity surfaces. The predictions made by these properties are compared to experimental and computed adsorption energies of two nucleophilic probe molecules, i.e. the Lewis bases $\mathrm{CO}$ and $\mathrm{H}_{2} \mathrm{O}$. Both $E(\mathbf{r})$ and $V(\mathbf{r})$ are obtained at the 0.001 a.u. isodensity

\footnotetext{
${ }^{a}$ Applied Physical Chemistry, Department of Chemistry, CBH, KTH Royal Institute of Technology, SE-100 44 Stockholm, Sweden. E-mail: tore@kth.se, joakim.halldin-stenlid@fysik.su.se

${ }^{b}$ Department of Physics, AlbaNova University Center, Stockholm University, SE-106 91 Stockholm, Sweden

${ }^{c}$ Swedish Nuclear Fuel and Waste Management Co (SKB), Evenemangsgatan 13, Box 3091, SE-169 03, Solna, Sweden

$\dagger$ Electronic supplementary information (ESI) available. See DOI: 10.1039/ с9ср03099a
}

surfaces, an approach which has been found useful for predictions and understanding of the interactive behavior of isolated molecules as well as transition metal and oxide nanoparticles. ${ }^{1-8}$

Through the analysis of these properties, we have previously been able to rationalize e.g. the enhanced catalytic behavior of $\mathrm{Au}$ nanoparticles; undercoordinated sites (e.g. corner sites) are associated with a depletion of electron density (known as a $\sigma$-hole) and a corresponding local maximum in $V(\mathbf{r}) .{ }^{4}$ The $\sigma$-holes are more pronounced at the corner sites than at terrace sites; this explains the stronger interactions at corners, which are necessary for an increased catalytic activity compared to the inert, perfect surfaces of Au. We have found that the interactions at undercoordinated $\mathrm{Au}$ sites are similar in origin to those of e.g. hydrogen and halogen ${ }^{9}$ bonding. A similar behavior is also found for $\mathrm{Ag}$ and $\mathrm{Cu}$ nanoparticles. ${ }^{7}$ In light of these observations, we have introduced a new type of bonds, regium bonds, ${ }^{7}$ that take place between the $\sigma$-hole of a neutral $\mathrm{Au}, \mathrm{Ag}$ or $\mathrm{Cu}$ site and a Lewis base, e.g. $\mathrm{CO}$ or $\mathrm{H}_{2} \mathrm{O}$. Striving to explore similarities and differences within chemical interaction behavior over the periodic table, we will herein investigate if the above concepts are more generally applicable and can be used to also explain interaction tendencies of extended surface as well as of other metals and materials.

The use of DFT-based descriptors to characterize the reactive properties of surfaces is a common practice in the surface science community. An important example is the d-band model of Hammer and Nørskov, ${ }^{10}$ which have been successfully 
employed to rationalize trends in adsorption energies on transition metal substrates and thereby, in the extension, to predict e.g. catalytic activity of metals and alloys. ${ }^{11,12}$ Compared to the traditional descriptors, including the d-band model, both $E(\mathbf{r})$ and $V(\mathbf{r})$ have the advantage of being local in their nature. This means that they can not only be used to analyze the difference in reactivity/activity between different materials, but also the variation in reactivity over the same surface, i.e. the regioselectivity. In addition, we have found that the $E(\mathbf{r})$ and $V(\mathbf{r})$ are able to predict the local adsorption behavior for the interaction of Lewis bases with oxide surfaces; a process for which there are no generally applicable descriptor, including the d-band model and its extensions. ${ }^{13-15}$ In the following we will demonstrate that local minima in $E(\mathbf{r}), E_{\mathrm{S}, \min }$, and local maxima in $V(\mathbf{r}), V_{\mathrm{S}, \max }$, on isodensity contours indicate sites susceptible towards interaction with electron donating molecules. We will also show that the relative magnitude of $E_{\mathrm{S} \text {,min }}$ and $V_{\mathrm{S}, \max }$ closely reflects trends in adsorption energies.

\section{Theory}

We shall begin by readopting the formulism of the descriptors to fit the solid-state framework. For a polyatomic non-periodic, isolated system (e.g. a gas phase molecule), $V(\mathbf{r})$ at position $\mathbf{r}$ is rigorously defined by

$$
V(\mathbf{r})=\sum_{A} \frac{Z_{A}}{\left|\mathbf{R}_{A}-\mathbf{r}\right|}-\int \frac{\rho\left(\mathbf{r}^{\prime}\right) \mathrm{d} \mathbf{r}^{\prime}}{\left|\mathbf{r}^{\prime}-\mathbf{r}\right|}
$$

where $R_{A}$ and $Z_{A}$ are the position and charge of the $A$ :th atomic nuclei, and $\rho\left(\mathbf{r}^{\prime}\right)$ is the electron density function. The first of these terms comes from the ionic contribution to the electrostatic potential and the second term is the local Hartree potential. In the non-periodic framework, the vacuum potential of a free electron in its ground state is zero on an absolute scale $\left(V_{\mathrm{vac}}^{\mathrm{abs}}=0\right)$. Due to the periodic representation of standard planewave codes, infinity is implied in three dimensions. Therefore the electrostatic potential $\left[V^{\mathrm{per}}(\mathbf{r})\right]$ obtained from a periodic calculation is ill-defined and will be arbitrarily shifted by a constant $\Delta V$ relative to the absolute scale: ${ }^{16}$

$$
V^{\text {per }}(\mathbf{r})=V^{\mathrm{abs}}(\mathbf{r})+\Delta V
$$

$V^{\text {per }}(\mathbf{r})$ will, furthermore, be shifted to different relative scales depending on the considered system; different atomic compositions, structures, orientations and vacuum distances results in different shifts. ${ }^{17,18}$ For the comparison of $V(\mathbf{r})$ [and $E(\mathbf{r})$ ] from one surface to another, a prerequisite is the usage of a common scale. Herein we will (approximately) shift $V(\mathbf{r})$ back to absolute numbers by comparison to the potential $V_{\mathrm{vac}}^{\text {per }}$ at the mid-point of the vacuum region between two slabs (illustrated in Fig. 1a). ${ }^{17-20}$ In summary:

$$
V^{\mathrm{abs}}(\mathbf{r})=V^{\mathrm{per}}(\mathbf{r})-V_{\mathrm{vac}}^{\mathrm{per}}
$$

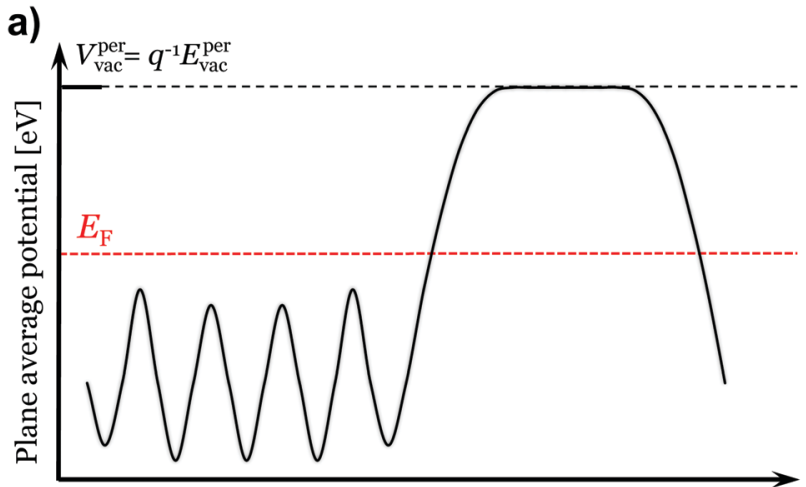

Lattice position in surface normal direction $[\AA]$

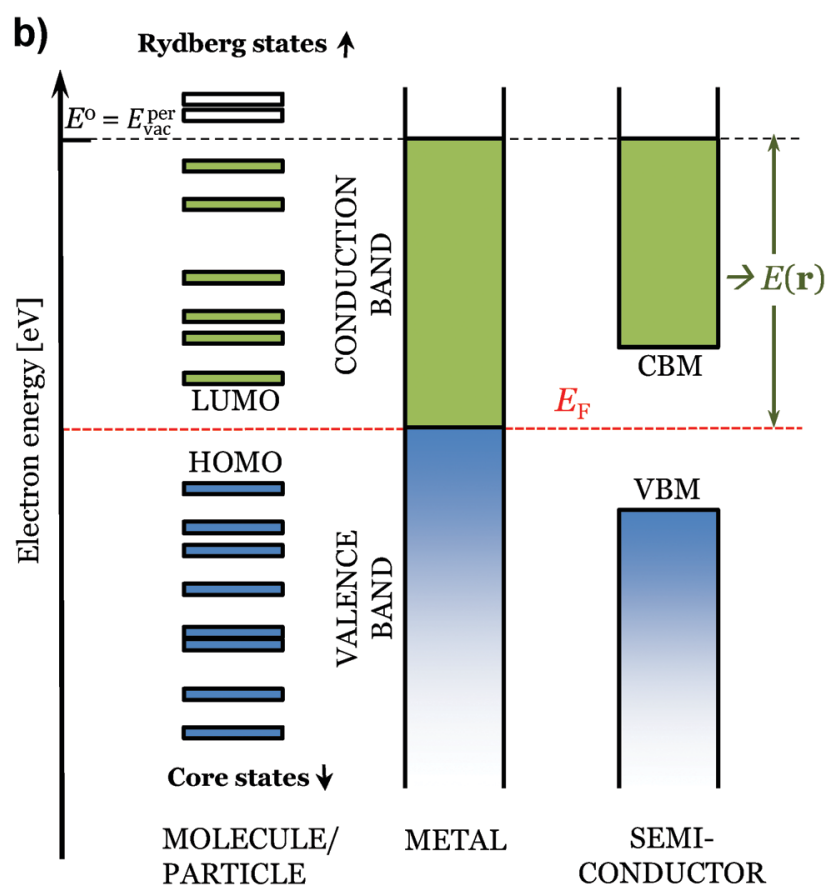

Fig. 1 In (a) plane average electrostatic potential over a periodic slab. In (b) illustration of the considered states for the evaluation of $E(\mathbf{r})$ of different types of compounds.

Hence $V(\mathbf{r})$ will here be determined as:

$$
V(\mathbf{r})=\sum_{A} \frac{Z_{A}}{\left|\mathbf{R}_{A}-\mathbf{r}\right|}-\int \frac{\rho\left(\mathbf{r}^{\prime}\right) \mathrm{d} \mathbf{r}^{\prime}}{\left|\mathbf{r}^{\prime}-\mathbf{r}\right|}-V_{\text {vac }}^{\text {per }}
$$

Turning next to the $E(\mathbf{r})$ property, we define it as (based on its definition for isolated systems ${ }^{2}$ )

$$
E(\mathbf{r})=\sum_{\varepsilon_{i}>E_{\mathrm{F}}}^{E_{0}} \frac{\left(\varepsilon_{i}-E_{0}\right) \rho_{i}(\mathbf{r})}{\rho(\mathbf{r})}
$$

to fit the solid-state framework. Here $\rho_{i}(\mathbf{r})$ and $\varepsilon_{i}$ are the density function and eigenvalue of the $i$ :th electronic state, including contributions from all virtual (unoccupied) one electron states going from the Fermi level, $E_{\mathrm{F}}$, up to a certain energy off-set, $E_{0}$. On an absolute scale, and within exact generalized Kohn-Sham 
DFT (GKS-DFT), the logical choice of $E_{0}$ is zero. ${ }^{2}$ This follows directly from Janak's theorem, ${ }^{21}$ which stipulates that the addition of an (partial) electron to any one-electron state above $\varepsilon_{i}=0$ corresponds to a net increase in energy. This would thus yield an unbound state that does not contribute to an attractive chemical interaction. Using the same reasoning as for the electrostatic potential, $E_{0}$ can be identified as the energy of an electron in the vacuum region of a periodic calculation, i.e. $E_{0}=E_{\mathrm{vac}}^{\mathrm{per}}$. Since $E=q V$, and since the charge of an electron is -1 in atomic units, it follows that $E_{\mathrm{vac}}^{\mathrm{per}}=-V_{\mathrm{vac}}^{\mathrm{per}}$. In a periodic framework the evaluation of $E(\mathbf{r})$ should also run over the entire k-space. Thus eqn (5) adapts to the form

$$
E(\mathbf{r})=\sum_{k=1}^{n_{\mathrm{kpt}}} \sum_{\varepsilon_{i}>E_{\mathrm{F}}}^{\varepsilon_{i}<E_{\mathrm{vac}}^{\mathrm{per}}} \frac{w_{k}\left(\varepsilon_{i, k}-E_{\mathrm{vac}}^{\mathrm{per}}\right) \rho_{i, k}(\mathbf{r})}{\rho(\mathbf{r})}
$$

where $n_{\mathrm{kpt}}$ is the number of k-points and $w_{k}$ is a symmetryweighting factor accounting for the number of irreducible representations of the studied k-point (see also Fig. 1b). For a spin-polarized calculation, the above summation runs over both the $\alpha$ and $\beta$ spin-states. $\neq$

$V(\mathbf{r})$ and $E(\mathbf{r})$ are complementary properties to a certain degree; whereas the $V(\mathbf{r})$ provides information on the electrostatic tendencies upon interaction, the $E(\mathbf{r})$ property also contains components related to charge-transfer and polarization. $E(\mathbf{r})$ can be partitioned into (shown here for the single k-point case): $:^{2,22}$

$$
E(\mathbf{r})=\frac{1}{\rho(\mathbf{r})} \sum_{\varepsilon_{i}>E_{\mathrm{F}}}^{\varepsilon_{i}<E_{\mathrm{vac}}}\left(\left[\frac{t_{i}(\mathbf{r})}{\rho_{i}(\mathbf{r})}-V^{\mathrm{per}}(\mathbf{r})-V_{\mathrm{XC}}(\mathbf{r})+V_{\mathrm{vac}}^{\mathrm{per}}\right] \rho_{i}(\mathbf{r})\right)
$$

where $t_{i}(\mathbf{r})$ is the local orbital kinetic energy density of the $i$ :th electronic state and defined as $t_{i}(\mathbf{r})=-1 / 2 \psi_{i}^{*}(\mathbf{r}) \nabla^{2} \psi_{i}(\mathbf{r}),{ }^{22}$ and $V_{\mathrm{XC}}(\mathbf{r})$ is the exchange-correlation potential. While $V_{\mathrm{XC}}(\mathbf{r})$ is approximately constant on an isodensity surface, $t_{i}(\mathbf{r})$ contains additional information as compared to $V(\mathbf{r})$ and is associated with the energy change of the system upon addition of electron density (i.e. charge-transfer). For the nucleophilic analog to $E(\mathbf{r})$, the average local ionization energy $\bar{I}(\mathbf{r})$ of Sjoberg et al., ${ }^{23,24}$ we propose the following expression for $\bar{I}(\mathbf{r})$ in the framework of periodic Kohn-Sham DFT (and Hartree-Fock):

$$
\bar{I}(\mathbf{r})=-\sum_{k=1}^{n_{\mathrm{kpt}}} \sum_{i=1}^{E_{\mathrm{F}}} \frac{w_{k}\left(\varepsilon_{i, k}-E_{\mathrm{vac}}^{\mathrm{per}}\right) \rho_{i, k}(\mathbf{r})}{\rho(\mathbf{r})}
$$

\section{Methods}

\section{Surface models}

To evaluate the descriptors, surface models where generated for different fcc metal substrates and for the transition metal oxides $\mathrm{Cu}_{2} \mathrm{O}$ and $\mathrm{TiO}_{2}$ in the anatase and rutile phases. The structures

\# We note that in future applications, for non-zero Kelvin interactions, a FermiDirac smearing of the electron occupation might be necessary to accurately capture the interaction behavior, thus leading to fractional occupation and corresponding weighting of the valence states. were based on crystal parameters from Xu et al. ${ }^{25}$ for the $\mathrm{TiO}_{2}$ structures, from Yu et al. ${ }^{26}$ for $\mathrm{Cu}_{2} \mathrm{O}$ and from Wellendorff et al. ${ }^{27}$ for the fcc metals. For $\mathrm{TiO}_{2}$ four-layer symmetric $(1 \times 1)$ unit cell slabs were used, separated by a $28 \AA$ vacuum distance, with the outermost layer being allowed to relax. A range of surface facets were studied including the anatase (100), (001) and (101), as well as rutile(100), (110) and (101). For $\mathrm{Cu}_{2} \mathrm{O}$, a six layer symmetric $(1 \times 1)$ unit cell slab model of the $(111)$ facet was used with the outer two layers free to relax using a vacuum separation of $24 \AA$. The $\mathrm{Cu}_{2} \mathrm{O}(111)$ surface with $1 / 3 \mathrm{ML} \mathrm{O}_{\text {Cus }}$ (unsaturated oxygen) vacancies was modeled by a $(\sqrt{ } 3 \times \sqrt{ } 3) R 30^{\circ}$ unit cell. $(1 \times 1)$ unit cells were employed for the fcc metal (111) surfaces using symmetric slabs comprising 10 layers with the three top layers free to relax and vacuum separations of $36 \AA$. For copper we evaluated the low-index (100), (110) and (111) surfaces as well as the stepped (221) and chiral (643) surfaces. This was also compared to the cuboctahedral $\mathrm{Cu}_{147}$ nanoparticle. For the remaining fcc metals, $\mathrm{Rh}, \mathrm{Ni}, \mathrm{Pt}, \mathrm{Pd}, \mathrm{Ir}, \mathrm{Ag}$, and $\mathrm{Au}$, only their (111) surfaces were included.

For comparison of site-specific interactions with $V(\mathbf{r})$ and $E(\mathbf{r})$, and in the cases when experimental data was not available, CO adsorption energies where determined for the different structures using $(2 \times 2)$ unit cells for the low index surfaces. The adsorption energies were calculated by:

$$
\Delta E_{\mathrm{ad}}=\left[E_{*_{\mathrm{CO}}}-\left(E_{*}+E_{\mathrm{CO}}\right)\right]
$$

where $E_{{ } \mathrm{CO}}, E_{*}$, and $E_{\mathrm{CO}}$ are the electronic energies of the adsorbate-surface, free surface, and gaseous CO, respectively. CO was studied in a $15 \times 15 \times 15 \AA^{3}$ unit cell.

\section{Computational details}

All calculations were carried out with the Vienna ab initio simulation package (VASP). ${ }^{28}$ The PBE xc-functional was used throughout. For the oxides, on-site $+U$ Hubbard corrections were included as proposed by Dudarev et al. ${ }^{29}$ The $(U-j)$ parameters were taken from Xu et al. ${ }^{25}$ for the $\mathrm{TiO}_{2}$ structures and from Yu et al. ${ }^{26}$ for $\mathrm{Cu}_{2} \mathrm{O}$. Grimme's D3 dispersion with BeckeJohnson damping was employed for the CO adsorption studies. $^{30,31}$ During optimization the core states were represented by standard PBE-PAW potentials. Valence electrons were represented by plane-wave basis sets with an energy cut-off of $400 \mathrm{eV} .4 \times 4 \times 1 \mathrm{k}$-point $\Gamma$-centered meshes were used for the sampling of the k-space during the geometry optimizations employing the tetrahedron method with Blöchl corrections ${ }^{32}$ for the oxides and the first order Methfessel-Paxton smearing method $^{33}$ for the metals. For the final energy evaluations and for the descriptor evaluations, $9 \times 9 \times 1$ meshes were used for the oxides and $11 \times 11 \times 1$ meshes for the metals. Hard PAW potentials and extended valences where used for the final energy and descriptor evaluations using a plane-wave cut-off of $800 \mathrm{eV}$. Molecular CO was treated by a single $\Gamma$-point. $V(\mathbf{r})$ where generated in VASP (LOCPOT including only the ionic and Hartree potential contributions). $E(\mathbf{r})$ was computed from the optimized wave function according to eqn (6) in the main article. Spin-polarization was considered for $\mathrm{Ni}(111)$. The $V_{\mathrm{S}}(\mathbf{r})$ and $E_{\mathrm{S}}(\mathbf{r})$ were visualized at the 0.001 a.u. $\left(\mathrm{e} \mathrm{Bohr}^{-3}\right)$ isodensity 
surface in VESTA. $^{34} V_{\mathrm{S} \text {, max }}$ and $E_{\mathrm{S} \text {, min }}$ where evaluated at the same isodensity surface.

\section{Results \& discussion}

Now to the central question: how do the $E(\mathbf{r})$ and $V(\mathbf{r})$ properties outlined above correlate with the Lewis acidic behavior of extended surfaces? The first feature that we shall explore is the local character of the descriptors. To investigate this, the properties are first mapped on the 0.001 a.u. isodensity contours of $\mathrm{Pt}(111), \mathrm{Cu}_{2} \mathrm{O}(111)$ and rutile $\mathrm{TiO}_{2}(110)$ shown in Fig. 2. These surface structures are suitable tests for the descriptors since the behavior upon adsorption of molecular species onto theses surfaces is experimentally well characterized.

On Pt(111) both the descriptors suggest that the sites on top of Pt atoms are the most susceptible to interactions with Lewis bases. This since $E_{\mathrm{S}, \min }$ and $V_{\mathrm{S}, \max }$ (also known as $\sigma$-holes ${ }^{9}$ ) appear and coincide on these sites, suggesting that the on top sites are the preferred sites based on both charge-transfer (i.e. low electron affinity as indicated by $E_{\mathrm{S}, \mathrm{min}}$ ) and electrostatic (i.e. strongly positive potential as indicated by $V_{\mathrm{S}, \max }$ ) arguments. Nucleophilic areas of low electrostatic potential are found at bridge and three-fold hollow sites in between atoms. Note again that $E_{\mathrm{S}}(\mathbf{r})$ can only be used to characterize local Lewis acidity whereas $V_{\mathrm{S}}(\mathbf{r})$ identifies both Lewis acidic $\left(V_{\mathrm{S}, \max }\right)$ and Lewis basic $\left(V_{\mathrm{S}, \mathrm{min}}\right)$ sites. The identified adsorption sites on $\operatorname{Pt}(111)$ agree well with the experimental findings that the Lewis basic CO adsorbs on top of Pt atoms. ${ }^{35-37}$ Standard DFT LDA or GGA calculations do, however, notoriously rank the CO affinities of on top and hollow site incorrectly - known as the CO-puzzle. ${ }^{38}$ Our results show that the on top site indeed is the preferred site with respect to electrostatics and the accepting of electron density from the sigma-orbital (HOMO) of CO. This in accordance with earlier studies, which attributed the preference for hollow sites

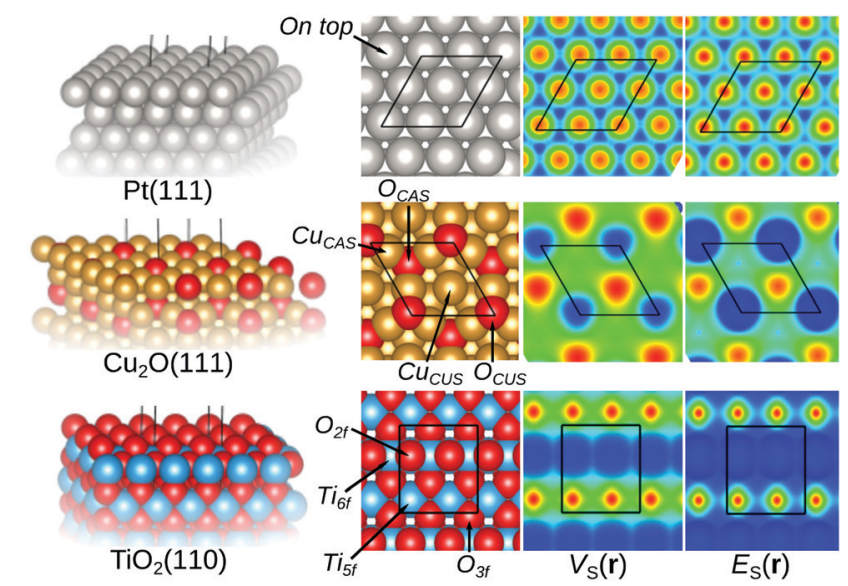

Fig. $2 E_{\mathrm{S}}(\mathbf{r})$ and $V_{\mathrm{S}}(\mathbf{r})$ mapped on the 0.001 isodensity contours for a selection of surface structures. For $E_{S}(\mathbf{r})$, Red $>$ yellow $>$ green $>$ cyan $>$ blue marks decreasing local electron affinity, i.e. decreasing Lewis acidy. For $V_{S}(\mathbf{r})$ red $>$ yellow sites are more positive (Lewis acidic), while blue $>$ cyan marks negative (Lewis basic) sites. Note, in particular, that metal atom/ion on top sites (here $\mathrm{Pt}, \mathrm{Cu}^{+}$, and $\mathrm{Ti}^{4+}$ ) are identified as Lewis acidic for all compounds. to an incorrect leveling of the CO HOMO/LUMO energies compared to the band structure of Pt resulting in an overestimation of the back donation to the CO LUMO orbital at the hollow sites. ${ }^{39,40}$

Fig. 2 also includes the examples of the stoichiometric and non-polar $\mathrm{Cu}_{2} \mathrm{O}(111)$ surface and the rutile $\mathrm{TiO}_{2}(110)$ surfaces. For $\mathrm{Cu}_{2} \mathrm{O}(111)$, the ideal surface contains two possible $\mathrm{Cu}$ adsorption sites, the coordinatively unsaturated $\mathrm{Cu}\left(\mathrm{Cu}_{\mathrm{CUS}}\right)$ and coordinatively saturated $\mathrm{Cu}\left(\mathrm{Cu}_{\mathrm{CS}}\right)$ and two $\mathrm{O}$ sites $\left(\mathrm{O}_{\mathrm{CUS}}\right.$ and $\mathrm{O}_{\mathrm{CS}}$ ). Among these, the $\mathrm{Cu}_{\mathrm{Cus}}$ site has been suggested as the favored site of interaction for Lewis bases, ${ }^{41}$ which has been confirmed by DFT calculations of e.g. CO, $\mathrm{H}_{2} \mathrm{~S}, \mathrm{H}_{2} \mathrm{O}$ and methanol adsorption. ${ }^{42-44}$ From the $E_{\mathrm{S}}(\mathbf{r})$ map of the surface, we find that the $\mathrm{Cu}_{\mathrm{CUS}}$ sites indeed correspond to local minima, while the $\mathrm{Cu}_{\mathrm{CS}}$ sites are attributed lower Lewis acidity, i.e. lower electron affinity. The $E_{\mathrm{S}}(\mathbf{r})$ indicates no tendency of $\mathrm{O}$ sites to be susceptible towards attack of Lewis bases. Hence, the predictions of the $E_{\mathrm{S}}(\mathbf{r})$ are in line with the established knowledge. $V_{\mathrm{S}}(\mathbf{r})$ outlines the same Lewis acidic surface characteristics as $E_{\mathrm{S}}(\mathbf{r})$, but also ranks the $\mathrm{O}_{\mathrm{CUS}}$ as more Lewis basic (more negative potential) than $\mathrm{O}_{\mathrm{CS}}$, again in agreement with the established picture. ${ }^{44,45}$ Fig. 3 shows the same $\mathrm{Cu}_{2} \mathrm{O}$ surface but with $1 / 2 \mathrm{ML} \mathrm{O}_{\mathrm{CUS}}$ vacancies $\left(\mathrm{V}_{\mathrm{O}_{\text {CUS }}}\right)$, a commonly observed surface reconstruction. ${ }^{46}$ This leaves behind a new type of undercoordinated $\mathrm{Cu}$ adsorption sites, $\mathrm{Cu} @ \mathrm{~V}_{\mathrm{O}_{\mathrm{Cus}}}$. Regardless, the most prominent $E_{\mathrm{S} \text {,min }}$ and $V_{\mathrm{S} \text {,max }}$ are still located at the same $\mathrm{Cu}_{\mathrm{CUS}}$ site as on the unreconstructed surface suggesting these sites remain the most favorable adsorption sites. Again, this is in line with previous understanding of e.g. $\mathrm{H}_{2} \mathrm{O}$ and methanol interactions onto this surface. ${ }^{44,45}$ From the above, we note that our methods can be used for comparison to e.g. experimental STM (scanning tunneling microscopy) images, as well as in the initial assessment of reactive sites of complex nanostructured surfaces.

Concerning the rutile $\mathrm{TiO}_{2}(110)$ surface, there are two possible Lewis acidic Ti sites, one six-fold $\left(\mathrm{Ti}_{6 \mathrm{f}}\right)$ and on fivefold $\left(\mathrm{Ti}_{5 \mathrm{f}}\right)$ coordinated. Similarly, there are two Lewis basic $\mathrm{O}$ sites, one three-fold $\left(\mathrm{O}_{3 \mathrm{f}}\right)$ and one two-fold coordinated $\left(\mathrm{O}_{2 \mathrm{f}}\right)$. $E_{\mathrm{S}}(\mathbf{r})$ and $V_{\mathrm{S}}(\mathbf{r})$ rank the $\mathrm{Ti}_{5 \mathrm{f}}$ site as more Lewis acidic (i.e. stronger electron affinity and higher positive potential) than the $\mathrm{Ti}_{6 \mathrm{f}}$ site, whereas the $\mathrm{O}_{2 \mathrm{f}}$ is more Lewis basic than $\mathrm{O}_{3 \mathrm{f}}$, in line with experimental and previous DFT results for e.g. $\mathrm{H}_{2} \mathrm{O}$, CO and methanol adsorption. ${ }^{47-50}$

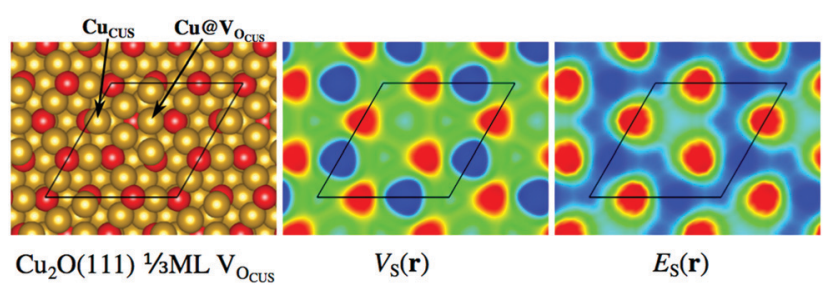

Fig. 3 Surface structure of $\mathrm{Cu}_{2} \mathrm{O}$ (111) with 1/3 $\mathrm{ML} \mathrm{O}_{\text {Cus }}$ vacancies to the left. The middle and right figures show $V_{S}(\mathbf{r})$ and $E_{S}(\mathbf{r})$ mapped on the 0.001 a.u. isodensity contour of the same $\mathrm{Cu}_{2} \mathrm{O}$ surface. The unsaturated $\mathrm{Cu}_{\text {Cus }}$ site is identified as the most electrostatically positive site as well as the site with highest electron affinity, in line with its strong Lewis acidity compared to other sites. 


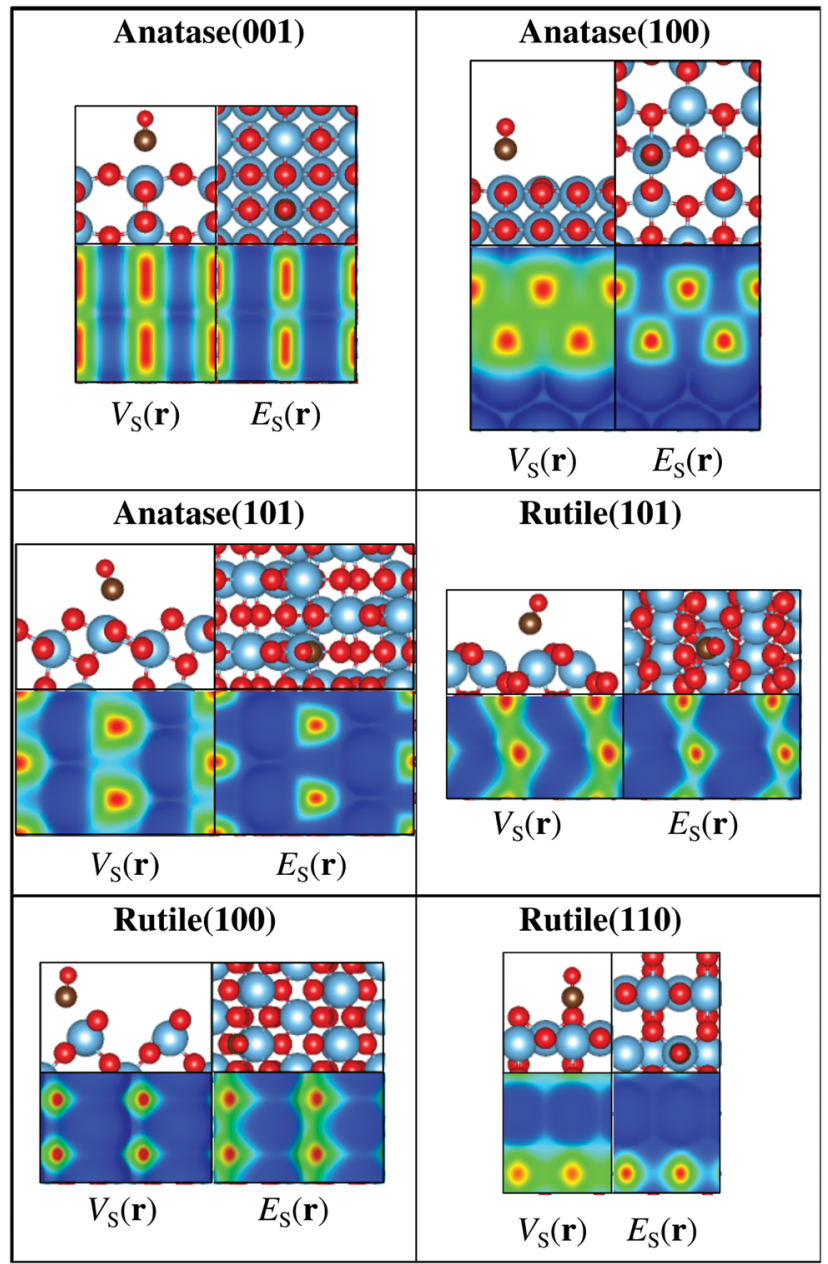

Fig. 4 Rutile and anatase $\mathrm{TiO}_{2}$ surfaces with $\mathrm{CO}$ adsorbed at its favored adsorption site as determined by DFT PBE-D3 calculations. Ti in light blue, $O$ in red, and $C$ in brown. Also shown are the 0.001 a.u. $E_{S}(\mathbf{r})$ and $V_{S}(r)$ isocontour maps of the surfaces. Lewis acidic sites (red $>$ yellow) are found close to the on top sites of $\mathrm{Ti}^{4+}$, and correspond well to the sites of CO adsorption. Note that for the anatase(101), rutile(101) and (100) surfaces, the adsorption of $\mathrm{CO}$ occurs slightly distorted from the on top site, which is well reproduced by the location of the $V_{S, \max }$ and $E_{S, \min }$.

In addition to rutile(110), we have also investigated the ability of the descriptors to identify $\mathrm{CO}$ adsorption sites on other $\mathrm{TiO}_{2}$ surface facets including the rutile(101) and (100), as well as the anatase(100), (101), and (001) facets. $\mathrm{TiO}_{2}$ is $e . g$. an important photocatalyst material, and these facets constitute the most commonly observed surfaces. ${ }^{51-53}$ Fig. 4 shows the above-mentioned $\mathrm{TiO}_{2}$ surfaces with $\mathrm{CO}$ adsorbed on the most favorable position and with the corresponding 0.001 a.u. $E_{\mathrm{S}}(\mathbf{r})$ and $V_{\mathrm{S}}(\mathbf{r})$ isocontour maps. Also for these surfaces it is found that the most extreme $E_{\mathrm{S} \text {,min }}$ and $V_{\mathrm{S} \text {, max }}$ correspond to the favored adsorption site.

From the above we conclude that $E(\mathbf{r})$ and $V(\mathbf{r})$ show promise as descriptors for the assessment of regioselectivity in surface Lewis acidity. However, the prevailing question is how well $E(\mathbf{r})$ and $V(\mathbf{r})$ can rank trends in adsorption energies for different substrates? Looking first at the series of $\mathrm{Cu}(111)$, (110) and (100) surface facets, Wang and Nakamura ${ }^{54}$ have determined their $\mathrm{H}_{2} \mathrm{O}$ affinities using GGA DFT as (110) $>(100)>$ (111). The $\mathrm{Cu}$ on top $E_{\mathrm{S}, \min }\left(V_{\mathrm{S}, \max }\right)$ for these surfaces are $-8.35 \mathrm{eV}$ $(0.44 \mathrm{eV}),-7.73(0.34)$, and $-6.53(0.33)$ thus reproducing the trends in the adsorption energies. The corresponding surface maps are shown in Fig. 5C-E.

We have previously used the local variations of $V_{\mathrm{S}}(\mathbf{r})$ to rationalize the enhanced catalytic activity of $\mathrm{Au}$ nanoparticles compared to its ideal crystalline surfaces. ${ }^{4}$ A similar analysis is here included for $\mathrm{Cu}$ using both $V_{\mathrm{S}}(\mathbf{r})$ and $E_{\mathrm{S}}(\mathbf{r})$, covering nanoparticles as well as ideal, stepped and kinked extended surfaces (Fig. 5). Analogous to $\mathrm{Au}$, we find that $\mathrm{Cu}$ nanoparticles display $V_{\mathrm{S}, \max }\left(\right.$ and $E_{\mathrm{S}, \mathrm{min}}$ ) on top of the exposed atoms, with the most prominent $V_{\mathrm{S} \text {,max }}$ and $E_{\mathrm{S} \text {,min }}$ at corner sites, followed by edge and, lastly, terrace sites (Fig. 5B). Similarly, the extended surfaces have the $V_{\mathrm{S}, \max }$ and $E_{\mathrm{S} \text {,min }}$ of largest magnitude (i.e. the most positive potential or strongest electron affinity) at corner sites followed by steps/edges, kinks (see definition in Fig. 5) and lastly terrace sites (Fig. 5C-G). Computed CO interaction energies follow the local descriptor values at the site of interaction. The correlations are strongest when comparing adsorption onto different sites for each substrate surface individually, but clear also when comparing adsorption onto all sites of all $\mathrm{Cu}$ substrates together (Fig. 5A). We note a tendency towards non-linear behavior going from the undercoordinated to the terrace sites. This could be seen as two different trends for the different classes of sites. We attribute this to a non-negligible effect of neighboring atoms, which could be captured by adding information from a larger coordination sphere to the interaction analysis.

One can also ask how sensitive the descriptors are to the choice of computational method? A test on $\mathrm{Cu}(100)$ using the BEEF-vdw ${ }^{55}$ DFT xc-functional instead of PBE corroborate the understanding from isolated systems ${ }^{2}$ that the $V(\mathbf{r})$ property is rather insensitive to the functional while the absolute values of $E(\mathbf{r})$ shifts depending on the choice of method. We find that $V_{\mathrm{S} \text {,max }}$ at the on top site is $0.34 \mathrm{eV}$ for both cases, whereas $E_{\mathrm{S} \text {,min }}$ is $-7.73 \mathrm{eV}$ at the PBE level of theory and $-7.45 \mathrm{eV}$ using BEEF-vdw. The behavior of the descriptors using different functionals will be further evaluated in a follow-up study.

We next assess the experimental CO adsorption enthalpies of a series of fcc (111) transition metal substrates. The adsorption enthalpies are taken from a database of Wellendorff et al. ${ }^{27}$ and includes the Ir, Rh, Ni, Pt, Pd and Cu surfaces. CO adsorbs on top of all but the Pd and Ni surfaces, where the hollow sites are preferred. ${ }^{27}$ Using the $E_{\mathrm{S} \text {, min }}$ value at the on top site from a 0.001 a.u. isodensity contour, we obtain an $R^{2}$ of 0.82 compared to the experimental adsorption enthalpies (Fig. 6 top). For CO, we obtain a slightly improved trend $\left(R^{2}\right.$ of 0.89$)$ when comparing to computed on top site adsorption energies at the PBE level§ and extending the series with $\mathrm{Au}$ and Ag. Fig. 6 bottom include data for computed $\mathrm{H}_{2} \mathrm{O}$ adsorption energies ${ }^{57}$ on fcc

$\S$ The CO adsorption energy bias of the PBE functional with respect to experimental data has here been accounted for by adding the corrections suggested by Petersen et al. ${ }^{56}$ to the computed values (i.e. a constant energy shift). No D3 corrections were used for this series. 


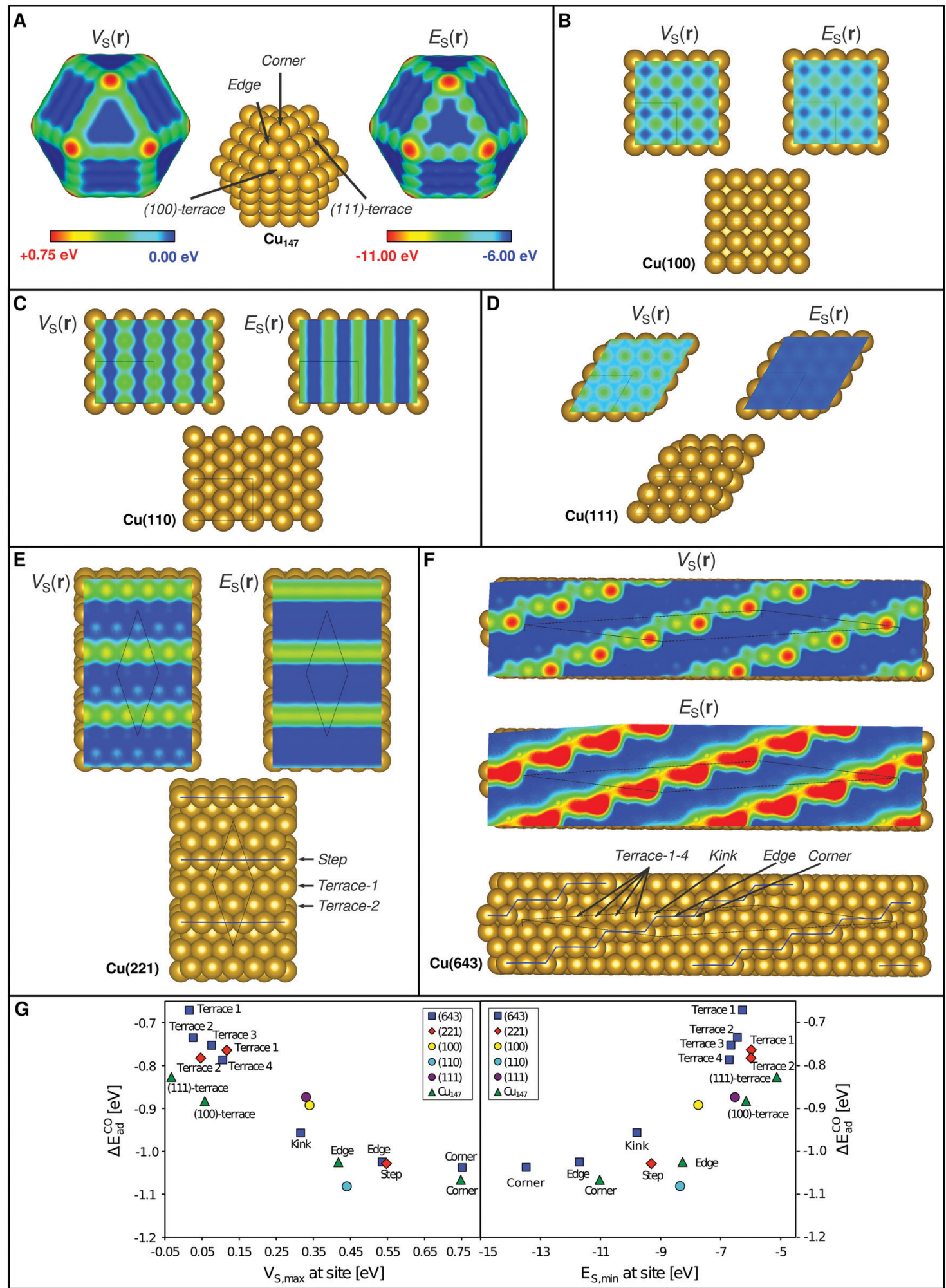

Fig. 5 Structure, $V_{S}(\mathbf{r})$ and $E_{S}(\mathbf{r})$ at the 0.001 a.u. isodensity surface for $(A)$ a copper cuboctahedral $\mathrm{Cu}_{147}$ nanoparticle, and in (B-F) for different crystallographic planes of $\mathrm{Cu}$. All images show the $V_{S}(\mathbf{r})$ and $E_{S}(\mathbf{r})$ properties on the common color scales depicted in (A). Similar images with scales optimized to display different adsorption sites on each individual compound are shown in Fig. S1 of the ESI. $\uparrow$ It can be noted that the more undercoordinated sites (corners > edges > kinks > facets) show larger positive electrostatic potential and higher electron affinity, which is found to correlate to a stronger Lewis acidity. In (G) CO interaction energies computed by DFT for the various sites of the different structures are compared to the local values of $V_{\mathrm{S}}(\mathbf{r})$ and $E_{\mathrm{S}}(\mathbf{r})$ (i.e. $V_{\mathrm{S}, \max }$ and $E_{\mathrm{S}, \mathrm{min}}$ ). 

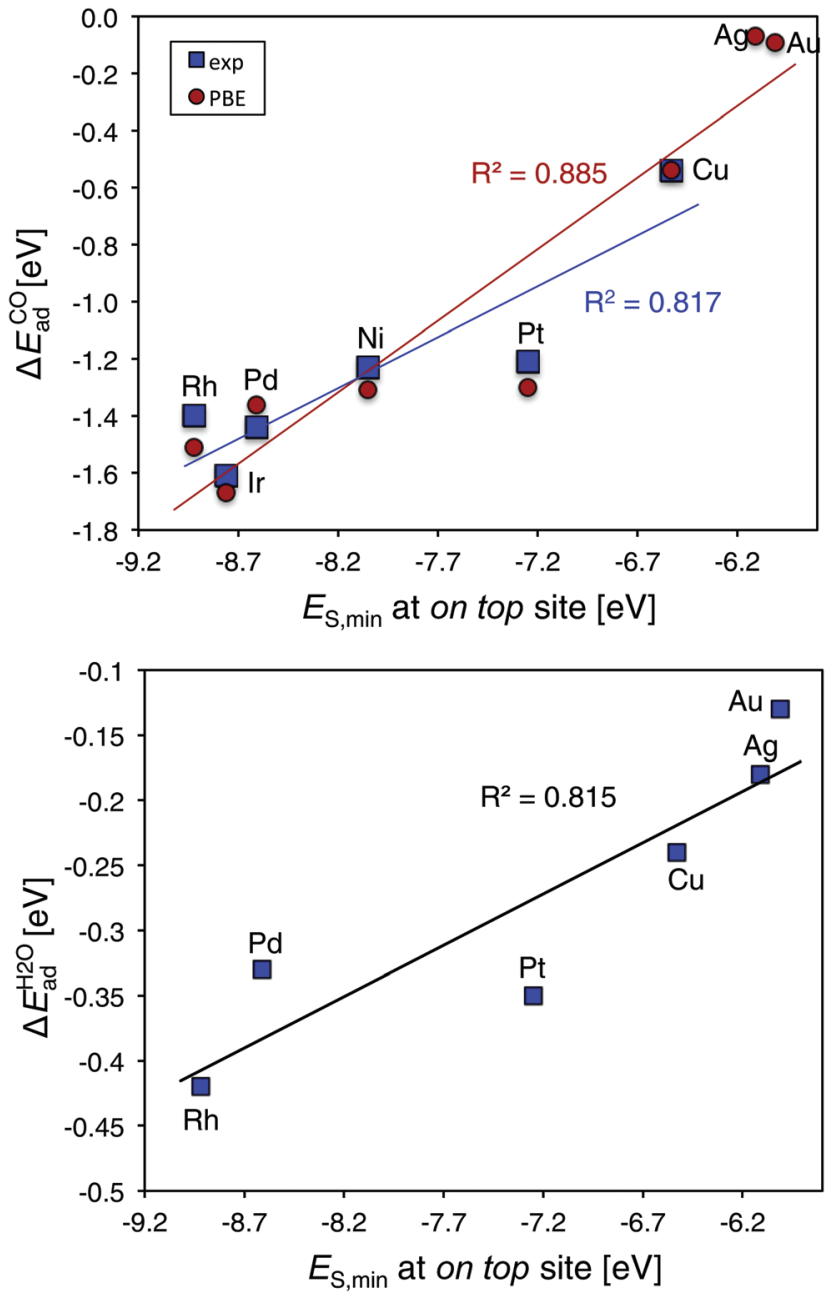

Fig. 6 Top, correlation plots $\mathrm{CO}$ adsorption energies ${ }^{27}$ and computed $E_{\mathrm{S}, \min } \S$ at the metal on top site of fcc TM(111) surfaces, TM $=\mathrm{Ir}, \mathrm{Rh}, \mathrm{Ni}, \mathrm{Pt}$ $\mathrm{Pd}, \mathrm{Cu}, \mathrm{Au}$, and $\mathrm{Ag}$. Bottom, computed $\mathrm{H}_{2} \mathrm{O}$ adsorption energies from Michaelides et al. ${ }^{57}$ versus $E_{\mathrm{S}, \mathrm{min}}$.

metals compared to $E_{\mathrm{S} \text {,min }}$ showing a correlation with an $R^{2}=0.82$. We note that the main outlier in all the series above is Pt.

The corresponding correlation of $V_{\mathrm{S}, \text { max }}$ versus experimental data is poor $R^{2}=0.16$. This suggests that the differences in CO affinity between the metal surfaces is determined by differences in charge-transfer capacity rather than electrostatics, which is in agreement with the success of the d-band model that downplays electrostatics in favor for orbital-band mixing.

Although the examples presented here represents a limited initial study, they demonstrate that the $E(\mathbf{r})$ and $V(\mathbf{r})$ descriptors can be used to predict and rationalize molecular interactions at both metal and oxide surfaces and have great potential for future applications. Results for other adsorbents including e.g. hydrogen atoms, hydrocarbons, water, ammonia and other nitrogen centered adsorbates will be presented in a follow-up study. For future directions, an interesting path is to further explore the Lewis basicity of metal and oxide surfaces. This could, as touched upon in the present study, be evaluated by the negative regions of $V(\mathbf{r})$ in combination with the Lewis basicity analog to $E(\mathbf{r})$, i.e. the $\bar{I}(\mathbf{r})$ of Sjoberg et al. ${ }^{23,24}$ This will be a focus of a future study.

\section{Conclusion}

In conclusion, we have shown that the local electron attachment energy $E(\mathbf{r})$, as well as the electrostatic potential $V(\mathbf{r})$, mapped on isodensity contours can reproduce the regional variations in Lewis acidity of metal and oxide surfaces. The relative magnitude at the site of interaction can, moreover, be used to correlate adsorption energy trends for $\mathrm{CO}$ and $\mathrm{H}_{2} \mathrm{O}$ onto fcc metal surfaces. A general applicability of the two properties for various types of surfaces and applications, e.g. in the fields of heterogeneous catalysis and electrochemistry, is envisioned. For instance, the approach shows great promise for the analysis of special sites/motifs on complex nanostructured material surfaces that may correspond to active sites for catalysis or sites that are susceptible to corrosion as well as seeding sites for electrochemical deposition. It is, moreover, likely that the descriptors can be used to screen for new, tailored materials of e.g. high catalytic activity, or for the comparison to experimental STM images as well as in the prediction of nanotoxicity by the differentiation of reactivity of nanoparticles. We furthermore recognize the applicability of our methods as a guiding tool for surface chemistry modeling, providing swift rationalization of the orientation and positioning of interacting molecules.

\section{Conflicts of interest}

There are no conflicts to declare.

\section{Acknowledgements}

This work was supported by the Swedish Nuclear Fuel and Waste management company (SKB) and the School of Chemical Science and Engineering at KTH (via its excellence award to JHS). JHS acknowledge financial support from the Aforsk foundation, and TB support from the Swedish Research Council (VR). The calculations were performed at resources provided by the Swedish National Infrastructure for Computing (SNIC) at the National Supercomputer Centre in Linköping University NSC as well as at the PDC Centre for High Performance Computing (PDC-HPC).

\section{References}

1 J. S. Murray and P. Politzer, The electrostatic potential: an overview, Wiley Interdiscip. Rev.: Comput. Mol. Sci., 2011, 1, 153-163.

2 T. Brinck, P. Carlqvist and J. H. Stenlid, Local Electron Attachment Energy and Its Use for Predicting Nucleophilic Reactions and Halogen Bonding, J. Phys. Chem. A, 2016, 120, 10023-10032. 
3 J. H. Stenlid and T. Brinck, Nucleophilic Aromatic Substitution Reactions Described by the Local Electron Attachment Energy, J. Org. Chem., 2017, 82, 3072-3083.

4 J. H. Stenlid and T. Brinck, Extending the $\sigma$-Hole Concept to Metals: An Electrostatic Interpretation of the Effects of Nanostructure in Gold and Platinum Catalysis, J. Am. Chem. Soc., 2017, 139, 11012-11015.

5 J. H. Stenlid, A. J. Johansson and T. Brinck, $\sigma$-Holes on Transition Metal Nanoclusters and Their Influence on the Local Lewis Acidity, Crystals, 2017, 7, 222.

6 J. H. Stenlid, A. J. Johansson and T. Brinck, Local Lewis Acidity of $\left(\mathrm{TiO}_{2}\right)_{n} n=7-10$ Nanoparticles Characterized by DFT-Based Descriptors: Tools for Catalyst Design, J. Phys. Chem. C, 2017, 121, 27483-27492.

7 J. H. Stenlid, A. J. Johansson and T. Brinck, $\sigma$-Holes and $\sigma$-lumps direct the Lewis basic and acidic interactions of noble metal nanoparticles: introducing regium bonds, Phys. Chem. Chem. Phys., 2018, 20, 2676-2692.

8 T. Brinck and J. H. Stenlid, The Molecular Surface Property Approach: A Guide to Chemical Interactions in Chemistry, Medicine, and Material Science, Adv. Theory Simul., 2019, 2, 1800149.

9 T. Clark, M. Hennemann, J. Murray and P. Politzer, Halogen bonding: the $\sigma$-hole, J. Mol. Model., 2007, 13, 291-296.

10 B. Hammer and J. K. Nørskov, Why gold is the noblest of all the metals, Nature, 1995, 376, 238-240.

11 T. Bligaard and J. K. Nørskov, in Chemical Bonding at Surfaces and Interfaces, ed. A. Nilsson, L. G. M. Pettersson and J. K. Nørskov, Elsevier, Amsterdam, 2008, pp. 255-321.

12 J. K. Nørskov, F. Studt, F. Abild-Pedersen and T. Bligaard, Energy Trends in Catalysis, in Fundamental Concepts in Heterogeneous Catalysis, John Wiley \& Sons, Inc, Hoboken, 2014, pp. 85-96.

13 H. B. Tao, L. Fang, J. Chen, H. B. Yang, J. Gao, J. Miao, S. Chen and B. Liu, Identification of Surface Reactivity Descriptor for Transition Metal Oxides in Oxygen Evolution Reaction, J. Am. Chem. Soc., 2016, 138, 9978-9985.

14 F. Calle-Vallejo, N. G. Inoglu, H.-Y. Su, J. I. Martínez, I. C. Man, M. T. M. Koper, J. R. Kitchin and J. Rossmeisl, Number of outer electrons as descriptor for adsorption processes on transition metals and their oxides, Chem. Sci., 2013, 4, 1245-1249.

15 J. K. Nørskov, F. Abild-Pedersen, F. Studt and T. Bligaard, Density functional theory in surface chemistry and catalysis, Proc. Natl. Acad. Sci. U. S. A., 2011, 108, 937-943.

16 L. Kleinman, Comment on the average potential of a Wigner solid, Phys. Rev. B: Condens. Matter Mater. Phys., 1981, 24, 7412-7414.

17 R. Ramprasad, P. von Allmen and L. R. C. Fonseca, Contributions to the work function: A density-functional study of adsorbates at graphene ribbon edges, Phys. Rev. B: Condens. Matter Mater. Phys., 1999, 60, 6023-6027.

18 C. Campañá, B. Mussard and T. K. Woo, Electrostatic Potential Derived Atomic Charges for Periodic Systems Using a Modified Error Functional, J. Chem. Theory Comput., 2009, 5, 2866-2878.
19 R. M. Feenstra and M. Widom, Low-energy electron reflectivity from graphene: First-principles computations and approximate models, Ultramicroscopy, 2013, 130, 101-108.

20 R. M. Feenstra, N. Srivastava, Q. Gao, M. Widom, B. Diaconescu, T. Ohta, G. L. Kellogg, J. T. Robinson and I. V. Vlassiouk, Lowenergy electron reflectivity from graphene, Phys. Rev. B: Condens. Matter Mater. Phys., 2013, 87, 041406.

21 J. F. Janak, Proof that $\partial E / \partial n_{\mathrm{i}}=\varepsilon_{\mathrm{i}}$ in density-functional theory, Phys. Rev. B: Condens. Matter Mater. Phys., 1978, 18, 7165-7168.

22 F. A. Bulat, M. Levy and P. Politzer, Average Local Ionization Energies in the Hartree-Fock and Kohn-Sham Theories, J. Phys. Chem. A, 2009, 113, 1384-1389.

23 P. Sjoberg, J. S. Murray, T. Brinck and P. Politzer, Average local ionization energies on the molecular surfaces of aromatic systems as guides to chemical reactivity, Can. J. Chem., 1990, 68, 1440-1443.

24 P. Politzer, J. S. Murray and F. A. Bulat, Average local ionization energy: A review, J. Mol. Model., 2010, 16, 1731-1742.

25 Z. Xu, J. Rossmeisl and J. R. Kitchin, A Linear Response DFT+U Study of Trends in the Oxygen Evolution Activity of Transition Metal Rutile Dioxides, J. Phys. Chem. C, 2015, 119, 4827-4833.

$26 \mathrm{~K}$. Yu and E. A. Carter, Communication: Comparing ab initio methods of obtaining effective $U$ parameters for closed-shell materials, J. Chem. Phys., 2014, 140, 121105.

27 J. Wellendorff, T. L. Silbaugh, D. Garcia-Pintos, J. K. Nørskov, T. Bligaard, F. Studt and C. T. Campbell, A benchmark database for adsorption bond energies to transition metal surfaces and comparison to selected DFT functionals, Surf. Sci., 2015, 640, 36-44.

28 G. Kresse and J. Furthmüller, Efficient iterative schemes for ab initio total-energy calculations using a plane-wave basis set, Phys. Rev. B: Condens. Matter Mater. Phys., 1996, 54, 11169-11186.

29 S. L. Dudarev, G. A. Botton, S. Y. Savrasov, C. J. Humphreys and A. P. Sutton, Electron-energy-loss spectra and the structural stability of nickel oxide: An LSDA + U study, Phys. Rev. B: Condens. Matter Mater. Phys., 1998, 57, 1505-1509.

30 S. Grimme, J. Antony, S. Ehrlich and H. Krieg, A consistent and accurate $\mathrm{ab}$ initio parametrization of density functional dispersion correction (DFT-D) for the 94 elements $\mathrm{H}-\mathrm{Pu}$, J. Chem. Phys., 2010, 132, 154104.

31 S. Grimme, S. Ehrlich and L. Goerigk, Effect of the damping function in dispersion corrected density functional theory, J. Comput. Chem., 2011, 32, 1456-1465.

32 P. E. Blöchl, O. Jepsen and O. K. Andersen, Improved tetrahedron method for Brillouin-zone integrations, Phys. Rev. B: Condens. Matter Mater. Phys., 1994, 49, 16223-16233.

33 M. Methfessel, High-precision sampling for Brillouin-zone integration in metals, Phys. Rev. B: Condens. Matter Mater. Phys., 1989, 40, 3616-3621.

34 K. Momma and F. Izumi, VESTA 3 for three-dimensional visualization of crystal, volumetric and morphology data, J. Appl. Crystallogr., 2011, 44, 1272-1276.

35 J. A. Stroscio and D. M. Eigler, Atomic and Molecular Manipulation with the Scanning Tunneling Microscope, Science, 1991, 254, 1319-1326. 
36 G. S. Blackman, M.-L. Xu, D. F. Ogletree, M. A. Van Hove and G. A. Somorjai, Mix of molecular adsorption sites detected for disordered $\mathrm{CO}$ on $\mathrm{Pt}(111)$ by diffuse low-energy electron diffraction, Phys. Rev. Lett., 1988, 61, 2352-2355.

37 H. Steininger, S. Lehwald and H. Ibach, On the adsorption of CO on Pt(111), Surf. Sci., 1982, 123, 264-282.

38 P. J. Feibelman, B. Hammer, J. K. Nørskov, F. Wagner, M. Scheffler, R. Stumpf, R. Watwe and J. Dumesic, The CO/Pt(111) Puzzle, J. Phys. Chem. B, 2001, 105, 4018-4025.

39 A. Gil, A. Clotet, J. M. Ricart, G. Kresse, M. GarciaHernández, N. Rösch and P. Sautet, Site preference of CO chemisorbed on $\mathrm{Pt}(111)$ from density functional calculations, Surf. Sci., 2003, 530, 71-87.

40 G. Kresse, Significance of single-electron energies for the description of CO on Pt(111), Phys. Rev. B: Condens. Matter Mater. Phys., 2003, 68, 073401.

41 A. Önsten, J. Weissenrieder, D. Stoltz, S. Yu, M. Göthelid and U. O. Karlsson, Role of Defects in Surface Chemistry on $\mathrm{Cu}_{2} \mathrm{O}(111)$, J. Phys. Chem. C, 2013, 117, 19357-19364.

42 W. An, A. E. Baber, F. Xu, M. Soldemo, J. Weissenrieder, D. Stacchiola and P. Liu, Mechanistic Study of CO Titration on $\mathrm{Cu}_{x} \mathrm{O} / \mathrm{Cu}(111)(x \leq 2)$ Surfaces, ChemCatChem, 2014, 6, 2364-2372.

43 J. H. Stenlid, A. J. Johansson, C. Leygraf and T. Brinck, Computational Analysis of the Early Stage of Cuprous Oxide Sulphidation: A Top-Down Process, Corros. Eng. Sci. Technol., 2017, 52, 50-53.

44 Z. Besharat, J. H. Stenlid, M. Soldemo, K. Marks, A. Önsten, M. Johnson, H. Öström, J. Weissenrieder, T. Brinck and M. Göthelid, Dehydrogenation of methanol on $\mathrm{Cu}_{2} \mathrm{O}(100)$ and (111), J. Chem. Phys., 2017, 146, 244702.

45 C. Riplinger and E. A. Carter, Cooperative Effects in Water Binding to Cuprous Oxide Surfaces, J. Phys. Chem. C, 2015, 119, 9311-9323.

46 A. Önsten, M. Göthelid and U. O. Karlsson, Atomic structure of $\mathrm{Cu}_{2} \mathrm{O}(111)$, Surf. Sci., 2009, 603, 257-264.
47 C. T. Campbell and J. R. V. Sellers, Enthalpies and Entropies of Adsorption on Well-Defined Oxide Surfaces: Experimental Measurements, Chem. Rev., 2013, 113, 4106-4135.

48 Y. Zhao, Z. Wang, X. Cui, T. Huang, B. Wang, Y. Luo, J. Yang and J. Hou, What Are the Adsorption Sites for CO on the Reduced $\mathrm{TiO}_{2}(110)-1 \times 1$ Surface?, J. Am. Chem. Soc., 2009, 131, 7958-7959.

49 B. Hammer, S. Wendt and F. Besenbacher, Water Adsorption on $\mathrm{TiO}_{2}$, Top. Catal., 2010, 53, 423-430.

50 K. Onda, B. Li, J. Zhao, K. D. Jordan, J. Yang and H. Petek, Wet Electrons at the $\mathrm{H}_{2} \mathrm{O} / \mathrm{TiO}_{2}(110)$ Surface, Science, 2005, 308, 1154-1158.

51 U. Diebold, The surface science of titanium dioxide, Surf. Sci. Rep., 2003, 48, 53-229.

52 P. Jones and J. A. Hockey, Infra-red studies of rutile surfaces. Part 2.-Hydroxylation, hydration and structure of rutile surfaces, Trans. Faraday Soc., 1971, 67, 2679-2685.

53 A. Vittadini, A. Selloni, F. P. Rotzinger and M. Grätzel, Structure and Energetics of Water Adsorbed at $\mathrm{TiO}_{2}$ Anatase (101) and (001) Surfaces, Phys. Rev. Lett., 1998, 81, 2954-2957.

54 G.-C. Wang and J. Nakamura, Structure Sensitivity for Forward and Reverse Water-Gas Shift Reactions on Copper Surfaces: A DFT Study, J. Phys. Chem. Lett., 2010, 1, 3053-3057.

55 J. Wellendorff, K. T. Lundgaard, A. Møgelhøj, V. Petzold, D. D. Landis, J. K. Nørskov, T. Bligaard and K. W. Jacobsen, Density functionals for surface science: exchange-correlation model development with Bayesian error estimation, Phys. Rev. B: Condens. Matter Mater. Phys., 2012, 85, 235149.

56 A. A. Peterson, F. Abild-Pedersen, F. Studt, J. Rossmeisl and J. K. Nørskov, How copper catalyzes the electroreduction of carbon dioxide into hydrocarbon fuels, Energy Environ. Sci., 2010, 3, 1311-1315.

57 A. Michaelides, V. A. Ranea, P. L. de Andres and D. A. King, General Model for Water Monomer Adsorption on ClosePacked Transition and Noble Metal Surfaces, Phys. Rev. Lett., 2003, 90, 216102. 